\title{
La formación docente universitaria en Cuba: Sus fundamentos desde una perspectiva desarrolladora del aprendizaje y la enseñanza*
}

\author{
University teaching training programs in Cuba: Fundamentals from learning \\ and teaching developing perspective \\ A formação de professores em Cuba: Seus fundamentos desde uma perspectiva \\ desenvolvimentista da aprendizagem e do ensino
}

\section{Diana Rosa Martín Sospedra. ${ }^{a}$}

a Universidad de Sancti Spíritus, Cuba. Fono: 53-4132383. Correo electrónico: dianarosa75@gmail.com

\begin{abstract}
RESUMEN
El continuo proceso de formación de los docentes en el escenario universitario cubano constituye una de las más altas prioridades del Ministerio de Educación Superior en el país, en correspondencia con los profundos cambios socio-culturales, económicos y tecnológicos, y sus crecientes demandas, desde el punto de vista de cómo alcanzar una mayor calidad en el aprendizaje de los estudiantes. En el presente trabajo se realiza un abordaje del proceso de formación del profesorado universitario, sus líneas directrices y fundamentos esenciales para la comprensión del aprendizaje y la enseñanza desde una perspectiva desarrolladora, que enfatiza la actuación pedagógica del profesor como mediador de la autonomía de sus estudiantes.
\end{abstract}

Palabras clave: formación docente, fundamentos, aprendizaje y enseñanza desarrolladores, educación superior.

\begin{abstract}
The continuous process of formation of professors at universities in Cuba is one of the highest priorities of the Ministry of Higher Education in the country, in correspondence with important socio-cultural, economic and technological changes, and increasing demands from the point of view of how to achieve a higher quality of learning on the students. In this project, there is an analysis of the process of formation of the professors, its guidelines and essential basics for the understanding of learning and teaching from a developer perspective, which emphasizes the professor's pedagogical activity as a mediator of the autonomy of their students.
\end{abstract}

Key words: formation of the professors, essential basics, developmental learning and teaching, higher education.

\section{RESUMO}

O contínuo processo de formação dos docentes no cenário universitário cubano constitui uma das mais altas prioridades do Ministério da Educação Superior no país, em correspondência com as profundas mudanças socioculturais, econômicas e tecnológicas e suas crescentes demandas, desde o ponto de vista de como alcançar

El texto forma parte de la investigación "La formación del profesorado universitario para la dirección del aprendizaje desde una perspectiva desarrolladora" que realiza la autora en la Universidade Federal de Uberlândia, bajo la orientación de la Dra. Andréa Maturano Longarezi y el financiamento de CAPES Foundation, Ministry of Education of Brazil, Brasília/DF Brazil, Programa CAPES/MES CUBA DOCENTES No. 009/2013 y el Conselho Nacional de Desenvolvimento Científico e Tecnológico - CNPq - Processo no. 405817/2012-6 e CAPES/OBEDUC - Projeto no. 11685. 
Estudios Pedagógicos XLI, N 1: 337-349, 2015

LA FORMACIÓN DOCENTE UNIVERSITARIA EN CUBA: SUS FUNDAMENTOS DESDE UNA PERSPECTIVA

DESARROLLADORA DEL APRENDIZAJE Y LA ENSEÑANZA

uma maior qualidade na aprendizagem dos estudantes. No presente trabalho, realiza-se uma abordagem do processo de formação do professorado universitário, suas diretrizes e fundamentos essenciais para a compreensão da aprendizagem e do ensino desde uma perspectiva desenvolvimentista, que enfatiza a atuação pedagógica do professor como mediador da autonomia de seus estudantes.

Palavras chave: formação docente, fundamentos, aprendizagem e ensino desenvolvimentista, educação superior.

\section{INTRODUCCIÓN}

El escenario social actual asiste a un continuo período de cambio que reclama con mayor exigencia conocimientos y destrezas a sus ciudadanos. Se es partícipe de una compleja revolución tecnológica, sustentada principalmente en la información y el conocimiento, este último con un papel determinante en el crecimiento económico y el bienestar social de los países. "Las ventajas competitivas de los países (o los sectores económicos) no pasan por la posibilidad de producir más unidades de producto, sino por la capacidad de introducir mayores conocimientos y tecnologías en los sectores productivos" (Pozner, 1997 Cit. en Álvarez y Fuentes, 2003). A nivel global se manifiesta una transición, donde la colocación del capital humano en el centro de las fuerzas que determinan la generación de riquezas, contribuye al logro de los ideales sociales, y las metas del desarrollo constituyen un pilar esencial en el crecimiento social.

Se precisa de nuevos aprendizajes y de la posibilidad de disponer de múltiples saberes alternativos en cualquier dominio del conocimiento humano, lo cual sitúa a la educación en la órbita de las prioridades políticas de los países. No es suficiente la acumulación de conocimientos por el individuo para la solución de problemas ante las nuevas situaciones de desarrollo, sino que se debe estar en condiciones de aprovechar y utilizar, durante toda la vida, cada oportunidad de actualización, profundización y enriquecimiento del primer saber, que posibilite la adaptación al cambio en la era del conocimiento (Núñez, Montalvo y Pérez, 2006). En ello las universidades desempeñan un rol fundamental al controlar la creación y la distribución de conocimientos, y erigirse como instituciones sociales indispensables para la formación de un número cada vez mayor de profesionales, además de que sirven con frecuencia como centros de pensamiento y acción política (Núñez, Montalvo y Pérez, 2007).

Estas instituciones de enseñanza superior han acompañado, en gran medida y desde hace décadas, el tránsito y el desarrollo del pensamiento social, y específicamente intelectual, cuyos enfoques y matices han sido diversos, en un primer momento y hasta la actualidad con predominio elitista, donde no se atesoran los conocimientos de forma privilegiada en la sociedad, ni es posible pensar en desempeños profesionales exitosos sin una adecuada y continua actualización. Esto va unido al tránsito gradual hacia niveles superiores de autonomía en el proceso de formación profesional, como condición necesaria para el logro de aprendizajes de calidad (MEC, 2003; TUNING, 2002).

Hoy la pertinencia de la universidad está estrechamente relacionada con la capacidad de adaptación a los contextos sociales y su condicionamiento, por lo cual requiere de una transformación para el logro de la eficacia en correspondencia con los profundos cambios socioculturales y económicos, que imprimen un sello particular a los sistemas educativos. No sólo se precisa de contribución en el plano de las ideas (currículos, análisis y evaluación de los sistemas educativos, desarrollo de la educación), sino también en la formación continua del profesorado. 
En este sentido, el panorama de la educación reinante es complejo desde el punto de vista de cómo alcanzar calidad en los aprendizajes de los estudiantes. Coexisten diferentes enfoques psicológicos que sustentan las pedagogías en uso, unos de amplio arraigo en las prácticas educativas, otros que tratan de imponerse como política educacional, y algunos distorsionados por el discurso modernista. ¿Qué rol puede y debe desempeñar la universidad en el perfeccionamiento de la calidad educacional y, por consiguiente, en el mejoramiento humano y el desarrollo social? ¿Cómo potenciar en este escenario una educación que conjugue excelencia y equidad, preparando a los educandos de hoy para vivir y trabajar en el mundo de mañana?

La experiencia acumulada por la autora, durante los últimos quince años en la educación superior relacionada con la carrera de Licenciatura en Psicología, ha posibilitado percibir que aún prevalece en los docentes una comprensión sobre el proceso de aprendizaje centrada esencialmente en los conocimientos, en un nivel reproductivo, memorístico, poco analítico, reflexivo y autorregulador, que no estimula en magnitud la formación de competencias profesionales, la creatividad, la adquisición de normas de comportamiento y métodos de aprendizaje con un efecto desarrollador, todo lo cual implica serias dificultades en la formación profesional. Los análisis realizados en los claustros de carrera, los colectivos de disciplina y el año académico, contribuyeron a la toma de conciencia de la existencia de una contradicción entre el modo en que se lleva a cabo el proceso de enseñanza-aprendizaje para la formación de los estudiantes, y las exigencias propias que la labor profesional les plantea.

Las situaciones de aprendizaje que se proponen a los estudiantes no siempre los motivan suficientemente, ni comprometen su trabajo intelectual hasta el punto de dejar una huella tanto en el plano de sus conocimientos, como en sus procesos de pensamiento y modos de actuación. Éstas generalmente son cerradas, no repercuten en los sistemas de clases de varias asignaturas, y pocas veces exigen que se trabaje de forma grupal, de modo que se pueda propiciar la comunicación, el planteamiento de interrogantes, conjeturas y la confrontación de puntos de vista. Unido a ello, no se aprovechan al máximo los conocimientos previos, las vivencias y las experiencias de los estudiantes, de entre los cuales un número considerable logra sólo con ayuda el establecimiento de nexos entre los fundamentos teóricos y prácticos en la solución de los problemas de la práctica profesional.

Ante los retos y realidades descritas anteriormente han surgido interrogantes, cuya conjugación armónica y coherente ofrece el hilo conductor para el abordaje del proceso de aprendizaje en el marco de la educación superior desde las perspectivas y demandas actuales de la pedagogía. ¿Cómo propiciar el desarrollo de la actividad intelectual productivocreadora y reflexiva, de modo que los estudiantes realicen aprendizajes de calidad y aprendan a aprender? ¿Cómo lograr que los aprendizajes adquieran un sentido personal para los estudiantes a partir del establecimiento de relaciones significativas de los nuevos contenidos con los anteriores, la experiencia práctica y el mundo afectivo individual? ¿En qué condiciones el proceso de enseñanza-aprendizaje contribuye al desarrollo de un fuerte sistema de motivaciones intrínsecas, autovaloraciones y expectativas positivas que orienten y sustenten la disposición y los esfuerzos de los estudiantes para aprender de manera activa, autónoma y permanente?

La respuesta a estas interrogantes exige, sin dudas, una reconceptualización del aprendizaje desde una posición epistemológica. Dicha reconceptualización debe revitalizar en la práctica educativa los postulados del enfoque histórico-cultural, línea directriz para la continua formación de los docentes universitarios. 
Estudios Pedagógicos XLI, N 1: 337-349, 2015

LA FORMACIÓN DOCENTE UNIVERSITARIA EN CUBA: SUS FUNDAMENTOS DESDE UNA PERSPECTIVA

DESARROLLADORA DEL APRENDIZAJE Y LA ENSEÑANZA

\section{DESARROLLO}

El perfeccionamiento continuo de la calidad educacional en correspondencia con el dinamismo de las demandas sociales contribuye a clarificar la misión de la universidad contemporánea hacia el cambio educativo en función del progreso. Esto bajo la premisa de que se produzcan logros de aprendizaje en términos de conocimientos, habilidades y valores que satisfagan los requerimientos de desempeño en la sociedad.

El cambio podría resumirse en la siguiente idea: el énfasis de la educación superior no sólo debe recaer sobre la transmisión de contenidos, sino en el proceso de aprehensión de los mismos más allá de un aprendizaje memorístico, que favorezca la apropiación activa y creadora de la cultura por parte de los individuos, ayudando al desarrollo de su autoperfeccionamiento constante, de su autonomía y autodeterminación, en íntima conexión con los necesarios procesos de socialización, compromiso y responsabilidad social (Ginoris, 2007; Zilberstein, 2004; Zilberstein y Silvestre, 2002; Castellanos, 2001). Desde este punto de vista, se precisa una transformación en el desempeño profesional pedagógico que conduce a la problemática de la educación de los educadores, al requerimiento de una cultura profesional que matice la expresión del quehacer cotidiano de los docentes en el marco de la dirección del proceso enseñanza-aprendizaje.

Cuba es un ejemplo en América Latina y en el resto del mundo en cuanto a su reforma universitaria y su sistema educativo, dado su alto grado de responsabilidad y disposición por parte de los actores involucrados al perfeccionamiento de la calidad educacional. En este contexto, la universidad, fruto de los innumerables esfuerzos de la Revolución Cubana durante más de cinco décadas, se consolida como entidad productora de conocimientos y con capacidad de proporcionar formación de alto nivel y comprometida con la sociedad, al tiempo que emerge como institución que lidera, en el orden teórico y práctico, el desarrollo social con la mayor integralidad, convirtiendo al país en uno de los más cultos en el ámbito internacional (Núñez et al., 2006a).

La nueva universidad cubana se dirige a preservar, desarrollar y promover toda la cultura de la humanidad, a través de procesos sustantivos y en estrecho vínculo con la sociedad, llegando con ello a todo el pueblo y, en particular, a los sectores más desfavorecidos. Entre sus características generales destaca su carácter científico, tecnológico y humanista, la labor educativa y político-ideológica, la formación investigativa de los estudiantes, y el vínculo del estudio con el trabajo, así como la universalización de la educación superior como rasgo distintivo que connota la riqueza conceptual e innovadora del sistema educativo en concordancia con las demandas sociales actuales.

Con la universalización, la universidad trascendió sus muros tradicionales y desarrolló sus procesos en estrecha interconexión con toda la sociedad, sobre una base fundamentalmente territorial. Todo esto se fundamenta en que la universidad es en esencia la sociedad misma, como un laboratorio natural con sus contradicciones y desarrollo, y, por lo tanto, abarca un espacio que no está constreñido a una instalación docente (Horrutinier, 2008).

Lo anterior trajo consigo una nueva mirada en la comprensión de los roles de profesores y estudiantes, con una reconceptualización muy ajena a cualquier concepto elitista y discriminatorio, pues se incluyen a todos los actores de este trascendental cambio en la formulación de una cultura de aprendizaje con igualdad de derechos y oportunidades para aprender a conocer, a ser, a hacer, a vivir juntos, a vivir con los demás, a desaprender lo obsoleto, De esta manera es posible responder, con la utilización armoniosa de la dialéctica, a las crecientes exigencias de la sociedad. 
Una de las consecuencias más directas del acceso universal al conocimiento en la sociedad informatizada es la necesidad de aprender con independencia. Junto con ello, se encuentra la necesidad de educación permanente a lo largo de la vida, con un carácter esencialmente autodirigido, en función de las particularidades individuales de cada persona, entendidas como la profesión, el contexto de desempeño, los cambios en el campo del conocimiento, entre otras. Emana de aquí el requerimiento pedagógico de enseñar a aprender sobre la base de prácticas que desarrollen la autonomía del educando, sustentadas en los fundamentos del enfoque histórico-culturalista, que acentúa el carácter desarrollador de la enseñanza y la función orientadora del profesor en el diseño de situaciones sociales de aprendizaje que conduzcan a la formación profesional del estudiante y su crecimiento como ser humano.

Orientar al estudiante en la construcción autónoma de conocimientos, habilidades, competencias, valores y actitudes profesionales plantea nuevas exigencias al docente universitario. Éstas superan el dominio de conocimientos y habilidades didácticas, en tanto el profesor precisa además de una motivación profesional intrínseca, de un compromiso moral con el ejercicio de la docencia, tanto en el aspecto práctico-profesional como teóricodidáctico, lo cual exige la continua formación del profesorado universitario (Cabrera, 2008; González, 2004, 2002; Imbernón, 2002, 2001, 1999, 1994).

La necesidad de sustituir las técnicas y estrategias de trasmisión-recepción por otras que orienten el aprendizaje como una tarea de indagación e investigación, que favorezcan la participación de los estudiantes en la construcción y reconstrucción de los conocimientos, resulta hoy un consenso global. Esto implica una formación en valores y una nueva actitud ante el saber, con el pleno ejercicio de la creatividad, la independencia pedagógica, la capacidad de autoperfeccionamiento, de investigación, y la capacidad para operar pedagógicamente con nuevas tecnologías.

A partir de este punto, la formación de los profesores ya no se realiza desde una concepción tradicional e instrumental por medio de cursos trasmisores de conocimientos y habilidades didácticas que posibilitan la enseñanza, sino como una formación en el marco de un proceso educativo potenciador del desarrollo del profesorado. Ésta transcurre de forma continua y matiza su desempeño competente, autónomo y comprometido en la dinámica cotidiana del docente.

La formación docente comprende todo un conjunto de procesos, a través de los cuales los profesores aprenden a aprender y a comprender, a analizar y a reflexionar en su labor diaria y, sin duda, a enseñar. Resulta imprescindible para la mejora del proceso educativo en la universidad, en continua evolución a partir de los cambios sociales, económicos, culturales y tecnológicos, que los docentes sientan la responsabilidad profesional y social de adquirir nuevas destrezas y conocimientos que optimicen tanto el quehacer diario en el despliegue del proceso docente-educativo, como el fomento de una cultura profesional.

Se concuerda con la profesora Viviana González Maura (2006) cuando relaciona la elevada y oportuna preparación del docente con la demanda de formar un profesional competente, pues todo proyecto de renovación que pretende elevar la calidad de la educación, reconoce la necesidad de involucrar en el cambio del docente su continua preparación y desarrollo, con el propósito de producir mejoras tangibles en la educación.

"Una educación y formación valiosa de los alumnos es prácticamente irrealizable al margen de una formación, también valiosa, relevante y efectiva de los profesores" (Escudero y Bolívar, 1994). "Quizás una de las claves del éxito en la dirección del cambio educativo sea entender que 
éste trata, ante todo, de la transformación del hombre, el maestro, y de hecho de los colectivos pedagógicos, tanto en su ser social como en su ser espiritual" (García y Addine, 2001). "Todo proceso de cambio, de reforma y de renovación pasa por los docentes, en cuanto a su aplicación en clases... La formación de los docentes está en el corazón de la evolución del sistema educativo" (Soussan, 2002).

Resulta un reto permanente para la educación superior cubana el perfeccionamiento del proceso de formación de los profesores, en su mayoría sin una formación pedagógica de base. La actuación pedagógica del profesor universitario como mediador de la autonomía de sus estudiantes demanda de él un alto nivel de formación pedagógica, didáctica y psicológica con la más alta cientificidad, lo cual requiere una continua preparación de los docentes. Ésta es caracterizada por la participación activa y reflexiva del profesor en su colectivo, dirigida tanto al desarrollo de aspectos cognoscitivos como del complejo componente afectivomotivacional, cuyo vínculo indisoluble posibilita un mejor desempeño, compromiso con la calidad y la ética en el ejercicio docente, lo que constituye, en efecto, el centro de atención de la investigación que se realiza.

Como proceso social y cultural, dicha formación obedece al carácter integral del desarrollo y la capacidad transformadora del individuo. Este proceso permite a cada sujeto la profundización en los contenidos socio-culturales, posibilitando saltos cualitativamente superiores en el desarrollo profesional y humano, a partir de la apropiación activa de la cultura, que acontece en la dinámica de la interacción con los otros y que promueve el desarrollo personológico del docente (Fuentes y Parra, 2008; Addine, 2004; García y Addine, 2003).

Constituye, a su vez, un producto de la interacción permanente, de la cooperación y la colaboración, del compromiso y el intercambio de experiencias y de conocimientos entre los sujetos del proceso: el profesorado. Tanto la comunicación como las interacciones personales que se llevan a cabo permiten la construcción de significados y sentidos, sobre la base de un actuar pedagógico que responde a los problemas de la práctica educativa cotidiana. Según Espinosa (2009), la socialización de los sujetos participantes y la difusión de la cultura pedagógica son la síntesis de la relación que se establece entre la preservación y la clara intención transformadora de la gestión de la formación.

En el marco de este proceso, el diagnóstico de las necesidades y las potencialidades de los docentes universitarios constituye el punto de partida para la implementación de las acciones formativas. El diagnóstico se relaciona con la sistematización de la experiencia cotidiana en la práctica, a partir de la discrepancia existente entre la situación actual y la deseada, resultante de la interpretación crítica de las regularidades que se dan en la práctica educativa (González y González, 2007).

De manera general, los modelos teóricos de formación docente universitaria abordan el proceso a partir del desempeño del profesional, centrados esencialmente en los sujetos y en sus relaciones, con énfasis en la calidad de los procesos universitarios (Parra, 2008; Guevara, 2006). En Cuba, la formación docente se sustenta desde un enfoque transformador del sujeto y su realidad, del profesor y el escenario universitario. La práctica educativa del profesor universitario se convierte en un espacio de desarrollo y progreso personal, que revela el nivel de desarrollo alcanzado en los ámbitos político-ideológico, docentemetodológico y científico-investigativo, los cuales posibilitan la consecución de cambios institucionales (Basora, 2007; González, 2004). 
La formación continua del profesorado universitario se lleva a cabo a través de las formas de superación contenidas en la actividad de postgrado: talleres, cursos, diplomados, maestrías y doctorados, y el trabajo metodológico. Este último se instituye como una de las vías más idóneas para la preparación docente.

El trabajo metodológico constituye un proceso dinámico y complejo, que en el contexto universitario logra atender las demandas concretas teórico-metodológicas, con el propósito de elevar el desarrollo profesional del profesorado en servicio y, por lo tanto, la calidad del proceso docente-educativo, centrado en la propia institución y sus sujetos, con resultados tangibles en el mejoramiento de la actividad docente. Es un proceso permanente de relación entre la teoría y la práctica pedagógica, consciente, sistemático y organizado, dirigido científicamente, participativo, multidisciplinar e integral.

La continua formación docente del profesorado posibilita evolucionar el contexto educacional en el que se actúa, como resultado del perfeccionamiento y la actualización de los contenidos, los métodos de la ciencia y sus valores en virtud de satisfacer las renovadas y crecientes exigencias de la educación superior. Los nuevos paradigmas educativos, como se apuntó anteriormente, se centran en el estudiante, sujeto activo y protagonista, que construye de manera muy creadora y personal el conocimiento (Zilberstein, 2004; Castellanos, 2001; Coll, 1992).

En este sentido, una concepción general sobre el aprendizaje representa una herramienta heurística indispensable que brinda una comprensión de los complejos y diversos fenómenos que tienen lugar en el aula, y por ende, un fundamento teórico, metodológico y práctico para planificar, organizar, dirigir, desarrollar y evaluar la práctica profesional, perfeccionándola continuamente. Todo ello constituye un requisito básico para que el educador pueda potenciar, de manera científica e intencional -y no empírica o intuitivamente- los tipos de aprendizajes necesarios, es decir, aquellos que propician en sus estudiantes el crecimiento y el enriquecimiento integral de sus recursos como seres humanos, en otras palabras, los aprendizajes desarrolladores (Baxter y Gil, 2002; Castellanos, 2001).

El aprendizaje resulta ser, en realidad, un proceso complejo, diversificado, altamente condicionado por factores tales como las características evolutivas del sujeto que aprende, las situaciones y los contextos socioculturales en los que aprende, los tipos de contenidos o aspectos de la realidad de los cuales debe apropiarse, y los recursos con los que cuenta para ello, el nivel de intencionalidad, conciencia y organización con que tienen lugar estos procesos, entre otros (Nieves, 2000). En relación con lo anterior, resultan presupuestos iniciales importantes para una comprensión del aprendizaje las siguientes consideraciones:

- Aprender es un proceso que ocurre a lo largo de toda la vida, y que se extiende en múltiples espacios, tiempos y formas, no es algo abstracto, está vinculado con las experiencias vitales, las necesidades de los individuos y con el contexto histórico-cultural concreto. En el aprendizaje se cristaliza continuamente la dialéctica entre lo histórico-social y lo individualpersonal; es siempre un proceso activo de reconstrucción de la cultura, y de descubrimiento del sentido personal y de la significación vital que tiene el conocimiento para los sujetos.

- Aprender supone el tránsito de lo externo a lo interno -en palabras de Vigotsky, de lo interpsicológico a lo intrapsicológico-, de la dependencia del sujeto a la independencia, de la regulación externa a la autorregulación. Supone el desarrollo cultural, esto es, recorrer un camino de progresivo dominio e interiorización de los productos de la cultura (cristalizados en los conocimientos, en los modos de pensar, sentir y actuar, y, también, de los modos de 
aprender), y de los instrumentos psicológicos que garantizan al individuo una creciente capacidad de control y transformación sobre su medio y sobre sí mismo (Febles y Canfux, 2006).

- El proceso de aprendizaje posee tanto un carácter intelectual como emocional; implica la personalidad como un todo. En él se construyen los conocimientos, destrezas, capacidades, se desarrolla la inteligencia, pero de manera inseparable. Este proceso es la fuente del enriquecimiento afectivo, donde se forman los sentimientos, valores, convicciones, ideales, donde emerge la propia persona y sus orientaciones ante la vida.

- Aunque el centro y el principal instrumento del aprendizaje es el propio sujeto que aprende, aprender es un proceso de participación, de colaboración y de interacción. En el grupo, en la comunicación con los otros, las personas desarrollan el auto-conocimiento, el compromiso y la responsabilidad, individual y social, elevan su capacidad para reflexionar divergente y creadoramente, para la evaluación crítica y la autocrítica, para solucionar problemas y tomar decisiones. El papel protagónico y activo de la persona no niega, en resumen, la mediación social.

Estas cuestiones permiten trascender la noción del estudiante como un mero receptor, un depósito o un consumidor de información, sustituyéndola por la de un aprendiz activo (e interactivo), capaz de realizar aprendizajes permanentes en contextos socioculturales complejos, de decidir qué necesita aprender en ellos, cómo aprender, qué recursos tiene que obtener para hacerlo y qué procesos debe implementar para obtener productos individual y socialmente valiosos (Betts, 1991). De aquí se deriva igualmente la noción de un aprendizaje eficiente y desarrollador.

Desde esta perspectiva, la continua formación de los docentes constituye una prioridad en la educación superior con vistas a perfeccionar la dirección del aprendizaje de los estudiantes. ¿Sobre cuáles fundamentos sustentar el proceso de formación de los docentes universitarios para la dirección del aprendizaje desde una perspectiva desarrolladora?

Desde el punto de vista filosófico, el proceso formativo de los docentes se basa en la concepción materialista de la historia a partir de los siguientes postulados: la concepción de la imagen del hombre como totalidad; la personalidad como conjunto de relaciones sociales en unidad de lo individual con lo social; el basamento filosófico de actividad como condición inherente al ser humano, quien conscientemente actúa sobre él mismo y sobre su entorno; la unidad dialéctica de la relación sujeto-objeto, que sufre la base de la mediación de la práctica, lo cual hace posible la interpretación recíproca de lo ideal y lo material en la actividad humana, y; el objeto como condicionante material y el sujeto como ser activo, consciente y transformador.

En correspondencia con lo anterior, la teoría del desarrollo histórico-cultural de la psiquis humana de Lev Semianovich Vygotski, eje central del enfoque histórico-cultural, constituye una significativa línea de base, pues reconoce el papel determinante tanto de la enseñanza como del aprendizaje en el proceso de culturalización del ser humano, siempre y cuando posea un efecto desarrollador sobre los mismos (Vygotski, 1987). Así, desde lo psicológico se asumen los postulados del enfoque histórico-cultural sobre la formación y el desarrollo de la personalidad como una totalidad biopsicosocial, matizada por la interconexión entre los componentes cognitivos, afectivos y conductuales.

En esta línea, la comprensión del aprendizaje deberá transitar de una definición unitaria a la aceptación de la existencia de múltiples aprendizajes, de su énfasis en el 
cambio de conductas a la visualización de la transformación constructiva y desarrolladora de la persona que aprende, de su ubicación en lo estrictamente individual a su redimensión socializadora. Asimismo, el papel del que aprende enfatizará la producción y la evaluación del conocimiento, se transfigurará de sumiso al poder del conocimiento a dominador del poder -que significa conocer-, de aspirar a la competencia como fin a emplearla como medio para aprender más, de sometido al control externo a poseedor de un comportamiento autorregulado.

Desde este enfoque, asumir una pedagogía desarrolladora implica concebir la educación dirigida al desarrollo integral de la personalidad de los estudiantes y de sus potencialidades, en particular, como resultado de la apropiación de la experiencia sociohistórica acumulada por la humanidad (Leontiev, 1975 Cit. en Febles y Canfux, 2006). Se es partidario de una enseñanza desarrolladora, que promueve un continuo ascenso en la calidad de lo que el estudiante realiza, vinculada inexorablemente a su crecimiento personológico. Esta enseñanza debe llegar a establecer coherentemente una unidad entre la instrucción, la educación y el desarrollo.

Por esto resulta imprescindible transformar la escuela actual, adoptar una nueva postura educativa de mayor alcance y potenciadora de valores culturales, sociales y morales. "La enseñanza desarrolladora debe trabajar no sólo por potenciar la "zona de desarrollo próximo" de cada estudiante, sino también actuar sobre la "zona de desarrollo potencial" del grupo al que pertenece" (Zilberstein, 2004). Las acciones formativas deben retomar el papel primario que tienen las acciones externas en el surgimiento y la formación de las acciones internas mentales. Aquí el concepto de orientación se convierte en piedra angular pues, de acuerdo a su calidad, dependerá la eficacia de la acción formada y, por tanto, del aprendizaje (Febles y Canfux, 2006; Nieves, 2000).

Desde un enfoque sociológico, la universidad como escenario para los aprendizajes por excelencia demanda la transformación de un espacio de reproducción a un espacio de creación, de un contexto de normativas a un contexto de participación, de un terreno de competencia a un terreno de cooperación, de un sitio centrado en el docente a un sitio centrado en las relaciones entre todos los actores que intervienen en la trama de aprender. A partir de lo anterior, el proceso formativo se basa en la individualización como proceso inseparable de la socialización, en el que el sujeto tiene como fundamento la actividad práctica de la cual se derivan las funciones cognitiva, valorativa y conductual de la personalidad.

El desarrollo del individuo, bajo la influencia de la educación y del medio social y natural, tiene lugar como una dialéctica entre objetivación y subjetivación de los contenidos sociales. La educación, como mediadora en los aprendizajes, debe cambiar de un medio para reproducir la sociedad a una palanca para el cambio social, de mecanismo cultural para asimilar al aprendiz a un mecanismo efectivo para que el aprendiz asimile la cultura, de proveedora de experiencias monoculturales a proveedora de experiencias multiculturales, de instrumento que promueve la uniformidad a facilitadora de la diversidad.

La historicidad de los procesos de aprendizaje rechaza los abordajes ahistóricos de la internalización como un proceso universal, pues, dada una comprensión relacional de la persona, el mundo y la actividad, la participación que constituye el núcleo de esta teoría del aprendizaje no puede ser internalizada como estructura de conocimiento, ni puede ser externalizada como artefactos o estructura de actividad general. La participación siempre está basada en negociaciones situadas y renegociaciones de significados en el mundo; 
esto implica que el conocimiento, la comprensión, y la experiencia están en constante interacción, por ende, son mutuamente constitutivas.

La noción de participación también disuelve las dicotomías entre cerebro, actividad cerebral y actividad del organismo como un todo, entre la contemplación y la implicación, entre la abstracción y la experiencia. En esta visión del aprendizaje, el conocimiento es entendido como algo inherente al crecimiento y la transformación de los sujetos, y está localizado en las relaciones entre éstos, su práctica, los instrumentos de su práctica, y la organización social, económica y política de los ámbitos de la práctica. Tal concepción sobre el aprendizaje determina cómo concebir, a su vez, el proceso de enseñar como una actividad cuyo polo dialéctico -el aprendizaje- establece en gran parte la manera en que ésta se debe estructurar (Addine, 2004).

Desde la arista pedagógica, se considera que la enseñanza ha de ser problematizadora y desarrolladora, concebir la didáctica desde el aprendizaje y no para el aprendizaje, el empleo de estrategias variadas como reconocimiento de las diferencias, el monitoreo del proceso más que la evaluación de productos, superar los programas cerrados regidos por la "ingeniería curricular" por programas abiertos sobre la base de los valores humanos. En este redireccionamiento, el rol del docente se centrará ya no como transmisor de saberes, sino como guía, conductor, promotor de la indagación y observador participante.

Se asume el requerimiento de organizar y estructurar el proceso de formación docente en relación con la vida, sostenido en las leyes de la pedagogía esbozadas por Álvarez de Zayas (1999). Esto es, la relación del proceso docente educativo con el contexto social -la escuela en la vida-, que establece el vínculo entre las necesidades sociales y la institución escolar, y la relación interna entre los componentes del proceso docente-educativo -la educación a través de la instrucción-.

La formación docente en el escenario universitario asume el sistema de principios pedagógicos propuesto por Addine (2002): la unidad del carácter científico e ideológico del proceso pedagógico; la vinculación de la educación con la vida, el medio social y el trabajo en el proceso de educación de la personalidad, y; la unidad de lo instructivo, lo educativo y lo desarrollador en el proceso de educación de la personalidad; la unidad de lo afectivo y lo cognitivo en el proceso de educación de la personalidad; el carácter colectivo e individual de la educación y el respeto a la personalidad; y la unidad entre la actividad, la comunicación y la personalidad. En apretada síntesis, se propone como punto de partida un conjunto de consideraciones básicas para el desarrollo de la formación del profesorado, que posibilita la expresión activa y protagónica de éstos en el despliegue de sus acciones, tales como: el carácter social de los objetivos, el enfoque orientador, la participación y el trabajo grupal, la interdisciplinariedad, aprender a aprender, y el rol de facilitador del docente en el proceso de enseñanza-aprendizaje.

Se asume una enseñanza desarrolladora como el proceso sistémico de trasmisión de la cultura en la institución escolar en función del encargo social, que se organiza a partir de los niveles de desarrollo actual y potencial de los estudiantes. Éste conduce el tránsito continuo hacia niveles superiores de desarrollo, con la finalidad de formar una personalidad integral y autodeterminada, capaz de transformarse y transformar la realidad en un contexto socio-histórico concreto.

En la búsqueda de alternativas desarrolladoras ha de tenerse en cuenta que una rigurosa y fundamentada creación, selección, organización, implementación y evaluación de estrategias a corto y largo plazo es un aspecto vital para garantizar la solución de los problemas 
y el logro de los objetivos propuestos para un aprendizaje desarrollador (Zilberstein, 2004). A su vez, la decisión sobre qué variantes introducir en la práctica educativa requiere de una concepción sistémica que permita tomar en consideración cómo se implican en ella cada uno de los componentes del proceso de enseñanza-aprendizaje (problema, contenido, objetivos, métodos, medios, evaluación) y sus principales protagonistas (estudiante-profesorgrupo).

Los puntos de vistas referidos hasta aquí constituyen una aproximación necesaria hacia los fundamentos sobre los cuales sustentar la formación continua de los docentes para la dirección del aprendizaje desde una concepción desarrolladora -del enseñar y el aprender-, presentados a partir de referentes filosóficos, psicológicos, filosóficos, y pedagógicos para su realización. Esto está a tono con el creciente y vertiginoso progreso científico-tecnológico actual, demandante de un esfuerzo cada vez mayor por parte de la escuela, particularmente en el escenario de la educación superior.

Hoy, como nunca antes, asistimos a un mundo laboral que requiere de una formación continua y un reciclaje profesional como consecuencia del cambiante mercado del trabajo en el que nos insertamos, un mercado flexible e incluso impredecible. Esto, unido al acelerado cambio de las tecnologías, obliga a los docentes a estar aprendiendo, de manera sistemática, algo nuevo.

\section{CONCLUSIONES}

La formación docente del profesorado en el escenario universitario cubano constituye una de las más altas prioridades de la educación superior en el país. Caracterizada por su papel transformador del docente y de éste como agente de cambio de la realidad educativa concreta, toma en consideración la experiencia profesional acumulada individualmente y la influencia del colectivo para la búsqueda de soluciones a los problemas más apremiantes del proceso docente-educativo y de las demandas sociales.

Tanto las formas de superación postgraduada como el trabajo metodológico constituyen la línea de base para la implementación de las acciones formativas. Lo anterior se concibe a partir del diagnóstico de las necesidades del claustro y las potencialidades para el perfeccionamiento de la preparación de los docentes, desde una perspectiva desarrolladora del aprendizaje y la enseñanza, que descansa en los postulados del enfoque históricocultural.

La formación docente se desarrolla durante toda la actividad profesional y, por su carácter diagnóstico, preventivo, permanente, flexible y desarrollador, ejerce una influencia determinante en el desempeño docente y en el mejoramiento del sistema educativo. Se trata de un proceso continuo de cambios evolutivos orientados a alcanzar etapas superiores de desarrollo en el desempeño del profesorado.

El análisis realizado con respecto a la dirección del proceso de aprendizaje acarrea una serie de consideraciones medulares acerca de la función de la universidad cubana y la capacidad de su personal docente para asumir la meta crucial de la calidad de los aprendizajes de los estudiantes, en relación con las demandas sociales actuales, cuya simiente basa la comprensión de la formación y desarrollo humano en la dialéctica materialista. Alcanzar verdaderos aprendizajes de calidad en la universidad conlleva una transformación sustancial y efectiva de la institución educativa, la cual no deberá 
Estudios Pedagógicos XLI, N 1: 337-349, 2015

LA FORMACIÓN DOCENTE UNIVERSITARIA EN CUBA: SUS FUNDAMENTOS DESDE UNA PERSPECTIVA

DESARROLLADORA DEL APRENDIZAJE Y LA ENSEÑANZA

confundirse con simples reestructuraciones, dadas sus implicaciones tanto psicológicas, sociológicas, como pedagógicas. En tal sentido, se requiere de alternativas que favorezcan la formación continua de los docentes universitarios en la dirección del aprendizaje desde una perspectiva desarrolladora.

El problema de la calidad de los aprendizajes, con gran fuerza en la educación superior, precisa de una atención más profunda y centrada en todos los actores del proceso. Para ello, la indagación teórica acerca de las raíces filosóficas, psicológicas, sociológicas y pedagógicas constituye una vía necesaria para allanar el camino que conduce a elevar los niveles de calidad en la formación integral de los docentes, en términos de conocimientos, habilidades y valores que satisfagan los requerimientos del desempeño en la sociedad, desde una educación que conjugue excelencia y equidad, basada en el perfeccionamiento educacional y, por consiguiente, en el mejoramiento humano y el desarrollo social.

\section{REFERENCIAS BIBLIOGRÁFICAS}

Addine, F. (2004). Didáctica: Teoría y práctica. La Habana: Pueblo y Educación. (2002). Principios para la dirección del proceso pedagógico. En G. Batista (Ed.), Compendio de pedagogía. La Habana: Pueblo y Educación.

Álvarez, B. y Fuentes, H. (2003). Didáctica del proceso de formación de los profesionales asistidos por la tecnología de la información y la comunicación. Santiago de Cuba.

Álvarez, C. (1999). La escuela en la vida. La Habana: Pueblo y Educación.

Basora, O. (2007). Modelo de gestión de la formación y de la identidad universitaria. Tesis de Doctorado en Ciencias Pedagógicas. Santiago de Cuba.

Baxter, E. y Gil, R. (2002). La escuela y el problema de la formación del hombre. En G. Batista (Ed.), Compendio de pedagogía. La Habana: Pueblo y Educación.

Betts, G. (1991). The Autonomous Learner Model for the Gifted and Talented. In N. Colangelo \& G. Davis (Eds.), Handbook of Gifted Education (pp. 142-153). Massachusetts: Allyn \& Bacon.

Cabrera, I. (2008). Desarrollo de la autonomía en el aprendizaje: Direcciones y estrategias en la formación profesional. Villa Clara: Centro de Estudios de la Educación UCLV.

Castellanos, D. et al. (2001). Hacia una concepción del aprendizaje desarrollador. La Habana: Instituto Superior Pedagógico Enrique José Varona.

Coll, C. (1993). Psicología y didáctica: Demarcación e interconexión. Infancia y Aprendizaje, (62-63), 237-243.

Espinoza, J. (2009). Gestión de la cultura profesional en la educación universitaria. Tesis de Doctorado en Ciencias Pedagógicas. Santiago de Cuba.

Febles, M. y Canfux, V. (2006). La concepción histórico-cultural del desarrollo. Leyes y principios. En L. Cruz (Ed.), Psicología del desarrollo. Selección de lecturas. La Habana: Félix Varela.

Fuentes, H. y Parra, A. (2008). La formación de los profesionales en la contemporaneidad. Santiago de Cuba.

García, G. y Addine, F. (2003). Profesionalidad y actividad investigativa del docente. Pedagogía 2003. Curso 54. Cuba.

Ginoris, O. (2007). El proceso de enseñanza-aprendizaje desarrollador. Componentes del proceso de enseñanza-aprendizaje: Objetivo, contenido y métodos de enseñanza-aprendizaje. Preparación Pedagógica para profesores en la nueva universidad cubana. Cuba.

González, R. y González, V. (2007). Diagnóstico de necesidades y estrategias de formación docente en las universidades. Revista Iberoamericana de Educación, (43), 6-15.

González, V. (2004). La investigación como eje transversal de la formación postgraduada del docente universitario. Cuarto Congreso Internacional de Educación Superior. La Habana, Cuba. 
(2004). El profesorado universitario: Su concepción y formación como modelo de actuación ética y profesional. Revista Iberoamericana de Educación, (12), 45-53.

. (2002). La profesionalidad del docente universitario desde una perspectiva humanista de la educación. Recuperado desde http://www.campus -oei.org/valores/boletin22.htm

Guevara, L. (2006). La gestión de la calidad del proceso de formación académica integral de los docentes de la Escuela de Trabajo Social de Mazatlán, Universidad Autónoma de Sinaloa. Tesis de Doctorado en Ciencias Pedagógicas. Santiago de Cuba.

Horrutinier, P. (2008). La universidad cubana: El modelo en formación. Estrategias de aprendizaje en la universalización. La Habana: Editorial Universitaria.

Imbernón, F. (2002). La investigación educativa como herramienta de formación del profesorado. Barcelona: Graò.

. (2001). La profesión docente ante los desafíos del presente y del futuro. En C. Marcelo (Ed.), La función docente (pp. 27-41). Madrid: Síntesis.

. (1999). Conceptualización de la formación y desarrollo profesional del profesorado. En

V. Ferreres y F. Imbernón (Eds.), Formación y actualización para la función docente (pp. 25-34). Madrid: Síntesis.

. (1994). La formación y el desarrollo profesional del profesorado. Hacia una nueva cultura profesional. Barcelona: Graò.

MEC (2003). La integración del Sistema Universitario Español en el EEES. Documento Marco. Madrid: MEC.

Nieves, Z. (2000). Aprendizaje desde el enfoque histórico cultural. Villa Clara: Centro de Estudios de la Educación UCLV.

Núñez, J., Montalvo, L. F. y Pérez, I. (2007). Universidad y desarrollo social basado en el conocimiento: Nuevas estrategias desde lo local. En A. Gallina, J. Núñez, V. Capecchi y L. F. Montalvo (Comps.), Innovaciones creativas y desarrollo humano (pp. 165-184). Montevideo: Trilce. . (2006a). La gestión del conocimiento, la información y la innovación tecnológica para el desarrollo local. En D. Hernández, F. Benítez, Y. Sánchez y S. Manzano (Comps.), La nueva universidad cubana y su contribución a la universalización del conocimiento (pp. 3-20). La Habana: Félix Varela.

. (2006b). Nueva universidad, conocimiento y desarrollo social basado en el conocimiento: Los marcos conceptuales del Programa Ramal, en busca de consensos. En J. Núñez y L. F. Montalvo (Eds.), Pensar ciencia, tecnología y sociedad (pp. 29-52). La Habana: Félix Varela.

Parra, A. (2008). Método para la formación permanente del docente de educación inicial del nivel preescolar del sector rural indígena, Apure, Venezuela. Tesis de Doctorado en Ciencias Pedagógicas. Santiago de Cuba.

TUNING (2002). Tuning Educational Structures in Europe. Retrieved from http://www.relint. Deusto.es/TUNING Project/spanish/doc2_fase1.asp

Vygotski, L. (1987). Historia del desarrollo de las funciones psíquicas superiores. La Habana: Científico Técnica.

Zilberstein, J. (2004). Aprendizaje desarrollador. Matanzas: Universidad de Matanzas. Educación. - y Silvestre, M. (2002). Hacia una didáctica desarrolladora. La Habana: Pueblo y 
